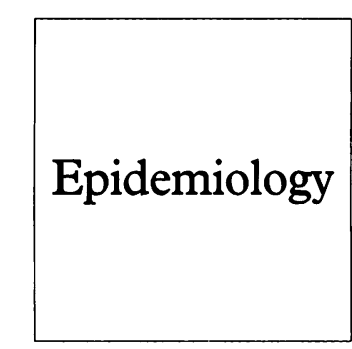

\title{
Epidemiology of gonococcal and chlamydial infections in Harrow and Brent
}

\author{
P Matondo, R Wall, K Morgan, M Hickman, C Dore, M Kapembwa
}

Objective: To describe the epidemiology and associated clinical features of gonorrhoea and chlamydial infection and to develop a profile of sexually transmitted diseases (STDs) in an outer London health district.

Design: Hospital-based retrospective study

Setting: Genitourinary medicine clinic, Northwick Park Hospital, Harrow and Brent Health Authority

Subjects: 70 male and female individuals with gonorrhoea and 129 with chlamydial infection, diagnosed consecutively over 28 months in 1992-94.

Results: More men than women had gonorrhoea (43 versus 27 ) but more women than men had chlamydial infection ( 84 versus 45 ), $\mathrm{p}<0.001$. There was a clear tendency for cases with either infection to locate along major road and rail transport routes. Foci of gonococcal infection were concentrated mainly in the densely populated areas, whereas chlamydial cases were more evenly spread. There was no significant effect of gender or type of STD on the odds ratio for residence in Harrow, single marital status or attendance for test of cure. However, the odds ratios for women having sexual intercourse with a regular partner only or previous STD were 5 (95\% CI $2 \cdot 4$ to 10.2$), \mathrm{p}<0.001$ and $0.3(95 \%$ CI 0.18 to 0.69$), \mathrm{p}=0.002$ times the odds for men, respectively. The odds ratios for patients with gonococcal infection being employed or having sex with a regular partner only were $0.5(95 \%$ CI 0.27 to 0.98$), p=0.04$ and $0.30(95 \% \mathrm{CI} 0.15$ to $0.60), p<0.001$ times the odds for patients with chlamydial infection, respectively. Of the women with gonorrhoea and previous pregnancy, $68 \%$ gave a history of abortion compared with $44 \%$ of those with chlamydial infection $(p=0.03)$.

Conclusion: The identification of foci of gonococcal and chlamydial infection and apparent location of these infections along the major transport routes in our health district require further study. That chlamydial infection, unlike gonorrhoea, is evenly distributed irrespective of population concentration and deprivation, suggests urgent need for a comprehensive local effort to control both STDs.

(Genitourin Med 1996;72:352-357)

Keywords: gonorrhoea; Chlamydia trachomatis; Harrow; Brent

\section{Introduction}

A specific target of the government's Health of the Nation strategy was to reduce the incidence of gonorrhoea among men and women aged 15-64 years by at least $20 \%$ by $1995 .^{1}$ In this strategy, gonococcal infection has been used as a surrogate marker of sexual health and the decreased incidence of gonorrhoea taken to indicate a reduced potential for transmission for HIV. Developing a local profile of sexually transmitted diseases (STDs) is an important first step in meeting this target since it defines the extent of the problem in the community and allows evaluation of risk profiles for effective intervention.

The aims of this study were two fold: firstly, to describe the epidemiology and other associated features of gonococcal and chlamydial infections in individuals attending the newly opened Genito-Urinary Medicine Clinic (GUM) at Northwick Park Hospital, Harrow, England. Secondly, since both Neisseria gonorrhoeae and Chlamydia trachomatis have short incubation periods, are curable and do not confer long lasting immunity, we sought to use the information obtained to develop a profile of STDs in our catchment population.

\section{Methods \\ Patient data}

The case notes of all individuals with gonococcal and chlamydial infections diagnosed in the GUM clinic at Northwick Park Hospital between April 1992 and July 1994 were retrieved and the following epidemiological and clinical information obtained from eligible patients: age, gender, sexual orientation, number of sexual partners during the last three months, area of residence including post code at presentation, employment status, previous history of STDs and outcome of treatment, marital and employment status. In addition, for females, parity and history of termination of pregnancy (TOP) and/or abortions were obtained. Written requests were sent out to neighbouring GUM clinics for information on the overall numbers of Harrow residents diagnosed with gonococcal or chlamydial infections in these units during the same period. Thirty-two such cases were reported from one clinic, 9 of whom had gonorrhoea and 23 chlamydial infection. These individuals including those attending ante-natal clinics or general practitioner surgeries were not included in this study due to inadequate information.

\author{
Address correspondence to: \\ Accepted for publication \\ 10 July 1996 \\ Department of \\ Medical Statistics, \\ Royal Postgraduate \\ Medical School
}


Detection of Neisseria gonorrhoeae and Chlamydia trachomatis

All persons attending sexually transmitted disease clinics in the department underwent testing for Neisseria gonorrhoea and Chlamydia trachomatis. Urethral and cervical swabs were taken using the microloop technique ( $1 \mu \mathrm{l}$ for men and $10 \mu \mathrm{l}$ for women) and inoculated directly on "gonococcal medium" (Modified New York City medium containing Columbia agar base, Unipath, Basingstoke, Hants UK) with $5 \%$ defibrinated lysed horse blood and the following antibiotics: lincomycin, colistin, trimethoprim and amphotericin B. Rectal swabs were obtained only in individuals practising anal sexual intercourse. Plates were incubated immediately in a humidified incubator at $37^{\circ} \mathrm{C}$ in an atmosphere of $5 \% \mathrm{CO}_{2}$ for up to 48 hours. Typical colonies of gram-negative cocci that were oxidase positive were identified as $N$ gonorrhoeae following carbohydrate utilisation tests. Culture remains the gold standard for the detection of $N$ gonorrhoeae with sensitivity ranging between $80 \%-95 \%{ }^{2}$

For men and women, urethral and cervical specimens, respectively were smeared directly on to slides and the presence of $C$ trachomatis demonstrated by staining for elementary bodies using direct immunofluorescence (DFA), (Syva MicroTrak, UK). Unlike gonorrhoea, there is no ideal method for detecting chlamydiae. However, the use of commercially-produced, fluorescein-conjugated monoclonal antibodies has found wide acceptance. This method is considered by some to be at least as sensitive as culture or PCR detection and more sensitive than enzymatic assays. ${ }^{3-5}$

\section{Analysis}

Data were analysed using Epi.Info and Stata statistical software packages. Fisher's exact test rather than the chi square test was used to determine whether there was an association between gender and each of the other variables because some of the categories had low frequencies. Logistic regression was then performed to predict the variable from two factors, sex of the respondent and STDs (gonorrhoea and chlamydial infection).

\section{Results}

Seventy cases of gonorrhoea ( 43 men and 27 women) and 129 cases of chlamydia (45 men and 84 women) were available for analysis. Analysis of patients' postcodes showed that most cases of gonorrhoea came from Harrow and Wembley (88\%). Of the neighbouring areas, Ruislip post codes accounted for $4 \%$ of cases only. The remainder, $8 \%$, either failed to give their address or offered post codes outside Harrow and adjoining areas (fig 1). For chlamydial infection, $84 \%$ of cases were resident in Harrow and Wembley. Ruislip accounted for $6 \%$ of cases. Ten per cent of individuals either withheld such information or lived outside the area (fig 2). Interestingly, there was an apparent tendency for cases with either infection to locate along the major road and rail transport routes; and clustering was seen in some areas. For example, two foci of gonococcal infection were identified in Wembley; one centred around a high density housing estate (Chalk Hill estate) and the other located in a residential unit near Wembley Stadium complex. Both areas lie at the intersection of two major roads and main line rail/underground transport systems linking Wembley to inner London districts as illustrated on the transport distribution map (fig 1). In contrast, cases of chlamydial infection appeared to be more evenly spread throughout the geographical area, although a chlamydial nidus was apparent in West Harrow.

Most of the gonococcal and chlamydial
Figure 1 Map of Harrow and part of Brent showing geographical location of cases of gonococcal infection in relation to major road/rail routes and Northwick Park Hospital. Harrow (includes Harrow Weald Wealdstone, Kenton, Edgware, Pinner, Brockley Hill and Stanmore). Brent (includes Wembley and Northwick Park Hospital)

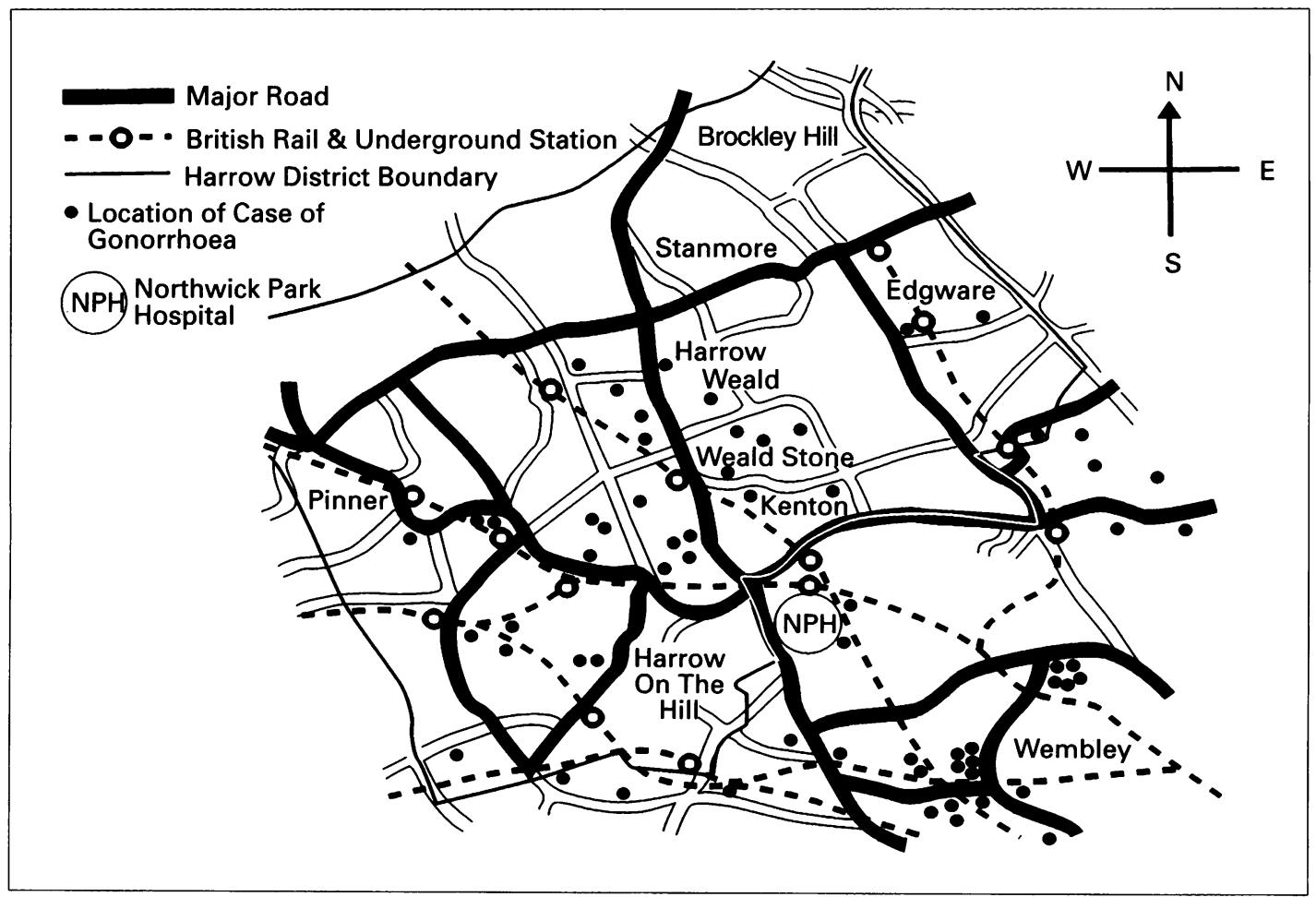


Figure 2 Map of Harrow and part of Brent showing geographical location of cases of chlamydial infection in relation to road/rail routes and Northwick Park Hospital. Harrow (includes Harrow Weald, Wealdstone, Kenton, Edgware, Pinner, Brockley Hill and Stanmore). Brent (includes Wembley and Northwick Park Hospital)

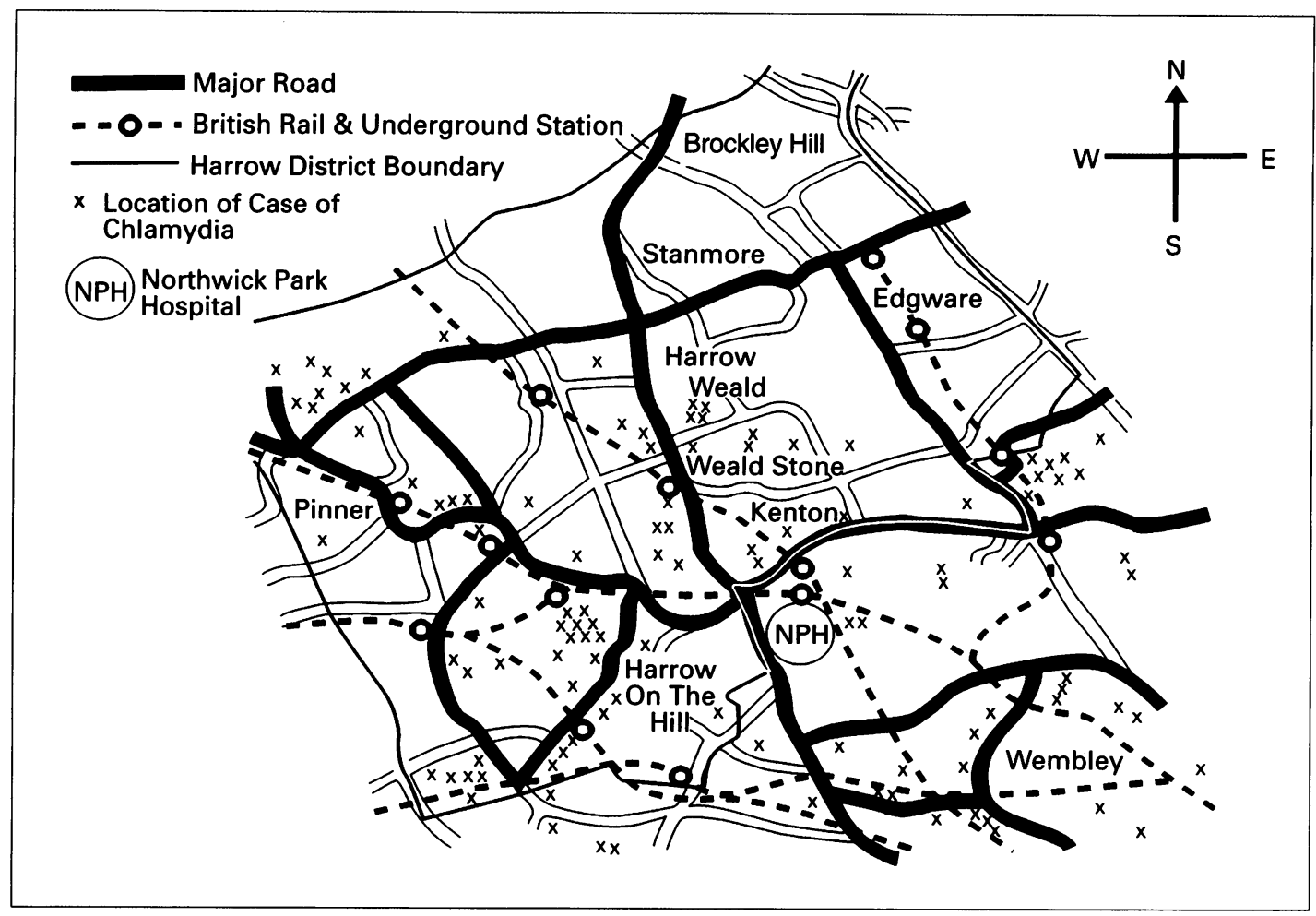

Table 1 Age and gender distribution of individuals with gonococcal $(n=70)$ and chlamydial $(n=129)$ infections in Harrow and Brent

\begin{tabular}{|c|c|c|c|c|}
\hline \multirow[b]{2}{*}{$\begin{array}{l}\text { Age } \\
\text { (years) }\end{array}$} & \multicolumn{2}{|c|}{ Gonorrhoea } & \multicolumn{2}{|l|}{ Chlamydia } \\
\hline & $\begin{array}{l}(n=70) \\
\text { Male }\end{array}$ & Female & $\begin{array}{l}(n=129) \\
\text { Male }\end{array}$ & Female \\
\hline $\begin{array}{l}<19 \\
20-24 \\
25-34 \\
35-44 \\
>45\end{array}$ & $\begin{array}{r}6 \\
15 \\
16 \\
6 \\
0 \\
43^{\star}\end{array}$ & $\begin{array}{r}7 \\
16 \\
3 \\
1 \\
0 \\
27\end{array}$ & $\begin{array}{r}4 \\
21 \\
18 \\
2 \\
0 \\
45\end{array}$ & $\begin{array}{r}16 \\
40 \\
22 \\
2 \\
4 \\
84^{\star}\end{array}$ \\
\hline
\end{tabular}

infections were acquired heterosexually (97\% and $96 \%$, respectively) and almost all were contracted from infected partners in the UK. Eight individuals had concomitant gonococcal and chlamydial infections. A history of recent casual sexual contact abroad was obtained in nine cases as follows: Thailand 4; Jamaica 2; Sweden 1; Portugal 1 and Africa 1.

Table 1 shows the age and sex distribution of cases of gonorrhoea and chlamydial infection. Both infections were detected at all age groups with the majority of infected individu- als in the 20-34 years group for both men and women. However, the sex ratios differed between the two infections ( $p<0.001)$ suggesting there might be a real discrepancy in exposure between the sexes to the two infections. The association between gender, and each of the major epidemiological features for both gonorrhoea and chlamydia as predicted from the logistic regression model is shown in table 2. The odds of women having sexual intercourse with one regular partner only was 5.0 times the odds for males $(p<0.001)$. The odds of individuals with gonorrhoea having sex with one regular partner only was 0.30 times the odds for those with chlamydial infection. This finding implies that patients with gonococcal infection were less likely $(p<$ 0.001 ) than those with chlamydial infection to have sex with a regular partner only.

There was no significant effect of gender or STD on the odds ratio for residence in Harrow, single marital status or having a test of cure performed $(p>0.05)$. The odds of patients with gonorrhoea being employed was

Table 2 Association between gender and selected risk factors for gonococcal $(n=70)$ and chlamydial $(n=129)$ infections as predicted by logistic regression

\begin{tabular}{|c|c|c|c|c|c|c|}
\hline \multirow{2}{*}{$\begin{array}{l}\text { Sexual or } \\
\text { demographic } \\
\text { characteristic }\end{array}$} & \multicolumn{3}{|c|}{ Gonorrhoea } & \multicolumn{3}{|c|}{ Chlamydia } \\
\hline & $\begin{array}{l}\text { Male } \\
(\%)\end{array}$ & $\begin{array}{l}\text { Female } \\
(\%)\end{array}$ & $\begin{array}{l}\text { Odds Ratio } \\
\text { (95\% CI) }\end{array}$ & $\begin{array}{l}\text { Male } \\
(\%)\end{array}$ & $\begin{array}{l}\text { Female } \\
(\%)\end{array}$ & $\begin{array}{l}\text { Odds Ratio } \\
(95 \% \text { CI })\end{array}$ \\
\hline Harrow residence & $24(71)$ & $17(81)$ & \multirow{6}{*}{$\begin{array}{l}1 \cdot 71 \\
(0 \cdot 48-6 \cdot 60) \\
1 \cdot 94 \\
(0 \cdot 47-8 \cdot 1) \\
0 \cdot 45 \\
(0 \cdot 16-1 \cdot 26) \\
4 \cdot 4 \dagger \\
(1 \cdot 5-12 \cdot 6) \\
0 \cdot 42 \\
(0 \cdot 14-1 \cdot 28) \\
2 \cdot 7 \\
(0 \cdot 96-7 \cdot 78)\end{array}$} & $35(83)$ & $61(75)$ & \multirow{6}{*}{$\begin{array}{l}0.61 \\
(0 \cdot 23-1 \cdot 59) \\
0 \cdot 84 \\
(0 \cdot 27-2 \cdot 59) \\
1 \cdot 33 \\
(0 \cdot 61-2 \cdot 93) \\
5 \cdot 5 \ddagger \\
(2 \cdot 0-14 \cdot 8) \\
0 \cdot 31 \star \\
(0 \cdot 13-0 \cdot 74) \\
0 \cdot 96 \\
(0 \cdot 4-2 \cdot 2)\end{array}$} \\
\hline Single Marital status & $33(80)$ & $24(89)$ & & $39(89)$ & $72(87)$ & \\
\hline Employed & $22(58)$ & $10(38)$ & & $25(61)$ & $52(68)$ & \\
\hline Regular partner only & $17(40)$ & $20(74)$ & & $30(67)$ & $77(92)$ & \\
\hline Previous STD & $17(41)$ & $6(23)$ & & $16(36)$ & $12(15)$ & \\
\hline Test of cure & $22(51)$ & $20(74)$ & & $33(73)$ & $61(73)$ & \\
\hline
\end{tabular}

${ }^{\star} \mathrm{p}=0.008 ; \mathrm{tp}=0.004 ; \neq \mathrm{p}<0.001$

( $95 \%$ CI): $95 \%$ Confidence Intervals. Previous STD: Includes history of previous gonorrhoea, chlamydia, trichomonas, herpes simplex virus, human papilloma virus infections or pelvic inflammatory disease. 
0.52 the odds for patients with chlamydial infection $(p=0.04)$ which suggests that the former were less likely to be in employment.

Twenty three $(34 \%)$ and $28(22 \%)$ of individuals with gonorrhoea and chlamydial infection respectively, gave a previous history of STD. A significant effect $(p=0.002)$ of gender on the odds of previous STD was seen. The odds of women having a previous history of STD was 0.35 times that for males. Individuals with gonorrhoea were more likely than those with chlamydial infection $(24 \%$ versus $4 \%$ ) to have a previous history of gonorrhoea within the last two years; and this difference was significant, $p=0.002$. In contrast, $4.5 \%$ and $4 \%$ of individuals with gonorrhoea and chlamydial infection respectively, gave a previous history of chlamydial infection. Furthermore, 18 of 27 women with gonorrhoea $(68 \%)$ had previous interrupted pregnancy namely, either termination or spontaneous abortion compared to 34 of 78 $(44 \%),(p=0.03)$ women with chlamydial infection.

\section{Discussion}

This study has identified foci of gonococcal and chlamydial infections in specific areas of a northwest London Health District (Harrow and Brent Health Agency). However, there are important limitations of the study which affect this conclusion. Firstly, only individuals attending one out-patient GUM clinic were studied and the analysis excludes individuals from our area attending neighbouring clinics or those presenting to obstetric/gynaecology units and general practitioners. The data are, therefore, incomplete and potentially underestimate the actual disease incidence as was shown in previous reports. ${ }^{67}$ Secondly, the sexual network patterns of infected individuals were not examined and therefore no distinction can be made between index cases and their contacts. Thirdly, the size of the populations at risk of infection in the affected geographical areas is not unknown. Finally, the accessibility and uptake of our STD services by people from various areas of the health district can not be ascertained with any accuracy.

The tendency of both infections to prevail along major transport routes is a phenomenon which, according to our knowledge, has not been described previously in this country. It is possible that such finding simply reflects population concentrations in these areas rather than greater health-seeking behaviour patterns or more successful contact tracing. However, the empirical association of a cluster of gonococcal infection and Wembley Stadium, a wellknown intertainment centre, suggests that availability of facilities conducive to the spread of sexually communicable infections is probably a major contributory factor to disease transmission. The distribution of the two infections is equally of interest since gonococcal infections were concentrated in the SouthEastern part of the district. This area has the highest population density with overcrowded households and relative deprivation. Ten- twenty per cent of the population in this area were defined as belonging to Social Classes IV/V in one survey whilst a major proportion of the rest of the population in Harrow (43\%) were assigned to Classes I and II. ${ }^{8}$ No similar observation was evident with respect to chlamydial infection which seemed to be diffusely spread over the catchment area.

A past history of gonorrhoea was a risk factor for re-acquisition of gonorrhoea. The presence of a minority of such individuals with repeat infection is indirect evidence supporting the hypothesis that gonococcal infection is neither randomly nor uniformly distributed and points to the existence of a subgroup of "core transmitters" in our catchment area. Although the size of this subgroup cannot be determined with accuracy from hospital data, such "repeaters" may constitute a small but distinct subgroup in the community substantiating the neighbourhood feature of gonococcal infection and distorting STD morbidity statistics, as noted by Lundin et al. ${ }^{9}$ Failure to identify and specifically target these subsets of individuals who continue to practice high risk behaviours may ultimately imperil our efforts to control STDs including HIV infection.

The above findings have implications for local public health interventions against the two infections. Firstly, subspeciation of gonococcal isolates can be used to track the emergence and spread of individual strains within the community as was shown by Handsfield ${ }^{10}$ in a cohort of intravenous drug using casual sex workers in Seattle, King Country, USA. In addition, the appearance within the community of newer antibiotic-resistant strains of $N$ gonorrhoeae can be monitored. Secondly, recognition of clusters of gonorrhoea and chlamydial infection allows us to undertake detailed analysis of behaviour and sexual network patterns of individuals with STD Thirdly, with respect to chlamydial data, intervention strategies need to be spread more widely across the catchment population in order to cater for this STD as well. In practice, this might imply targeting schools, colleges, well-women/men clinics and family planning clinics throughout the district rather than singling out "high risk areas" only.

Our results confirm other epidemiological studies from genitourinary clinics in England and Wales showing more men with gonorrhoea than women but more women than men with chlamydia. Given the biological similarities between gonococcal and chlamydial infection and the fact that both conditions tend to be asymptomatic in women, the disparity in the male-to-female ratio between the two infections shown in this study and from the national data for England and Wales ${ }^{112}$ is intriguing. A possible explanation is that diagnostic tests currently available for routine use detect chlamydia more readily in women than men. It has been suggested that non-cultura tests for chlamydia may be more sensitive in cervical than urethral infections because the number of chlamydia organisms is higher in the cervix than urethra. ${ }^{13}$ Studies on chlamydial testing place the sensitivity of DFA and 
ELISA in asymptomatic men at 50\%-84\%. ${ }^{14} 15$ If true, this would lead to under-diagnosis of chlamydial infection in men resulting in a spuriously low incidence in men on one hand, and increased infection of women by their untreated male partners, on the other. Alternatively, there might be greater efficiency for male-to-female transmission for chlamydia than gonorrhoea probably facilitated by a larger pool of asymptomatic chlamydial infection in men in the community. ${ }^{8}$ Studies involving male and female sexual contacts of patients with chlamydial infection, using culture $^{1617}$ or non-culture tests, ${ }^{18}$ have found a higher prevalence of chlamydia in female than male sexual contacts. The gender difference in the distribution of gonococcal infection is more difficult to explain. Since culture is the most sensitive method of detecting $N$ gonorrhoeae in both sexes, detection failure in females is unlikely to account for this discrepancy and it seems conceivable that higher female to male transmission rates occur in gonococcal infection. ${ }^{19} 20$

The relatively higher incidence of chlamydial infection in women, the high incidence of the same infection in England and Wales, in defiance of the conventional expectation of a decline in incidence similar to that observed with gonorrhoea and the mounting evidence suggesting that asymptomatic chlamydial infection in men rivals the proportions quoted for women ${ }^{16-21}$ argue for a re-think of current intervention strategies. Taylor-Robinson has recently pointed out the lack of a coherent and comprehensive screening programme in Britain. ${ }^{22}$ Epidemiological evidence suggests asymptomatic chlamydial infection among men in the community is important from the public health point of view. Interventions aimed at reducing the incidence of chlamydial infection ought, therefore, to consider ways of ensuring opportunistic screening of men in order to detect and treat asymptomatic chlamydial infection. Otherwise a pool of asymptomatic men who are probably more efficient transmitters of the infection than women, may maintain a high incidence of chlamydial infection in the community. ${ }^{21}$

The age distribution of individuals with either gonorrhoea or chlamydial infection was comparable and accords with that reported from other GUM clinics in England and Wales ${ }^{11223}$ although the proportion of teenagers with either infection in our study is smaller than has been reported nationally. ${ }^{24}$ However, there were more women aged under 25 years with chlamydial infection than gonorrhoea and this difference was significant, $p=$ $0 \cdot 015$. This finding is in keeping with national STD morbidity (KC60) ${ }^{11} 12$ data and underscores the need for specific behavioural and other interventions targeting these age groups. For example, Males ${ }^{25}$ has argued for a more forceful STD prevention programme in the USA which confronts, not only the excessive economic deprivation suffered by the young but also recognises the intrinsically integrated nature of sexual behaviours among adolescents and adults.
The strongly heterosexual mode of acquisition of infection is in contrast to that seen in clinics in central London where the proportion of homosexually acquired gonorrhoea in men is much higher. ${ }^{26}$ This finding might indicate a preference amongst gay men to attend the "traditional' GUM clinics rather than relative absence of infection in this client group in our district.

That $68 \%$ and $44 \%$ of women with gonorrhoea and chlamydial infection respectively, had previously interrupted pregnancy probably highlights a cohort of women who practice unprotected sexual intercourse without adequate contraception. Although a temporal association between termination of pregnancy TOP and STD infection cannot be inferred from our data, Blackwell et al have shown that a significant proportion of women undergoing TOP are infected with STD. ${ }^{6}$ These workers have also demonstrated health gains accruing from routine screening of all women for STD prior to TOP in terms of reduced post-abortal morbidity and cost-benefit impact. Thus, a case could be made for initiating similar intervention in our catchment population in collaboration with other relevant departments or agencies in pursuance of the Health of the Nation's objectives, ${ }^{1}$ if there was compelling evidence that similar benefits could be realised in our population.

\section{Conclusion}

The apparent location of foci of gonococcal and chlamydial infection along the major transport routes and differences in the distribution of the two STDs in this small scale study may have implications for local public health interventions. To what extent the data reflect the situation in the entire Brent and Harrow health district is difficult to determine. Further special surveys involving larger populations are required to monitor trends in particular population groups to assist progress towards achieving targets for sexual health including control of HIV infection.

We thank Professor David Taylor-Robinson and Dr Adrian Renton, St Mary's Hospital Medical School, Paddington, London, for their valuable comments and criticisms in the preparation of this manuscript

Department of health. The Health of the Nation. London. HMSO, 1992 (CM 1986).

2 Hook EW III, Hansfield HH. Gonococcal infections in the adult. In: Holmes KK, Mardh PA, Sparling PG et al. Sexually Transmitted Diseases. New York: McGraw-Hill; 1990:149-65.

3 Thomas BJ, Evans RT, Hawkins DA, Taylor-Robinson D. Sensitivity of detecting Chlamydia trachomatis elementary bodies in smears by use of a fluorescein-labelled monoclonal antibody: comparison with conventional chlamydial isolation. $\mathcal{F}$ Clin Pathol 1984;37:812-6.

4 Foulkes SJ, Deighton R, Feeney ARB, Mohanty KC Freeman CWJ. Comparison of direct immunofluorescence and cell culture for detecting Chlamydia trachomatis. Genitourin Med 1985;61:255-7.

5 Thomas BJ, MacLeod EJ, Taylor- Robinson D. Evaluation of the sensitivity of 10 diagnostic assays for Chlamydia trachomatis by use of a sample laboratory procedure. $\mathcal{F}$ trachomatis by use of a sam
Clin Pathol 1993;46:408-10.

6 Blackwell AL, Thomas PD, Wareham K, Emery SJ. Health gains from screening for infection of the lower genital tract in women attending for termination of pregnancy. tract in women attending
Lancet 1994;342:206-10.

7 Kudesia G, Zadik PM, Ripley M. Chlamydia trachomatis infection in males attending general practitioners. Genitourin Med 1994;70:356.

8 Vellani Z. All for health. Health for all in Harrow, Dept of Public Health Harrow Health Authority 1989:10-7. 
9 Lundin RS, Wright MW, Scatliff JN. Behavioural and social characteristics of the patient with repeated venereal disease and his effect on statistics on venereal diseases. $B$ IVenereal Dis 1977;53:140-4.

10 Handsfield HH. Recent developments in STDs. 1. Bacterial diseases. Hospital Practice 1991;26:47-56.

11 Sexually transmitted disease quarterly report: gonorrhoea in England and Wales. Communicable Disease Repor 1995;5:62-3.

12 Sexually transmitted disease quarterly report: genital infection with Chlamydia trachomatis in England and Wales. Communicable Disease Report 1995;5:122-3.

13 Stamm WE. Toward control of sexually transmitted chlamydial infections. Ann Interm Med 1993;119:432-4.

14 Shafer MA, Schachter J, Moncada J, et al. Evaluation of urine-based screening strategies to detect Chlamydia traurine-based screening strategies to detect Chlamydia tra-
chomatis among sexually active asymptomatic young chomatis among sexually active

15 Stamm WE. Diagnosis of Chlamydia trachomatis genitourinary infections. Ann Intern Med 1988;108:710-7.

16 Thelin I, Wennstrom A-M, Maardh P-A. Contact tracing in patients with genital chlamydia infection. $\mathrm{Br} \mathcal{F}$ Venereal Dis 1980;56:259-62.

17 Lycke E, Lowhagen G, Hallhagen G, Johannisson G, Ramstedt K. The risk of transmission of genital chlamydia trachomatis infection is less than that of genital Neisseria gonorrhoea infection. Sex Transm Dis 1980:7:6-10.

18 Matondo P, Johnson I, Sivapalan S. Morbidity and disease prevalence in male and female contacts of patients with genital chlamydial infections. Int $¥ S T D$ AIDS 1995;6: genital

19 Zelin JM, Robinson AH, Ridgway GL, Allason-Jones E, Williams P. Chlamydial urethritis in heterosexual men attending a genito-urinary medicine clinic: prevalence, symptoms, condom usage and partner change. Int $\mathcal{F} S T D$ AIDS 1995;6:27-30.

20 Singh G, Blackwell A. Morbidity in male partners of women who have chlamydial infection before termination of pregnancy. Lancet 1994;344:1438.

21 Karam GH, Martin DH, Flotte TR, et al. Asymptomatic Chlamydia trachomatis infections among sexually active men. F Infect Dis 1986;154:900-3.

22 Taylor-Robinson D. Chlamydia trachomatis and sexually transmitted disease: what do we know and what shall we to? $B M F$ 1994;308:150-1.

23 Blair I, Skett S Gon Communicable Disease Report 1994;4:R25-R28.

24 Department of Health New cases seen at genito-urinary Department of Health. New cases seen at genito-urinary
clinics in England. Summary information from KC60 1993-94; Tables 1 and 2. Government Statistical Service 25 Males AM. Adult involvement in teenage childbearing and STD. Lancet 1995;346:64-5

26 Renton AM, Ison CA, Whitaker L, Kirkland K, Kupek E Harris JRW. Neisseria gonorrhoeae isolated at St Mary's Hospital, London. 1980-91. Genitourin Med 1993;69: 286-9.

\title{
We hope you never
}

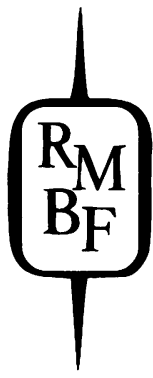

\section{need our help}

\author{
A nationwide support service \\ for doctors in need, \\ their wives, husbands and children
}

Help the medical profession help themselves

Donations and enquiries to:

The Secretary, Royal Medical Benevolent Fund

24, King's Road, Wimbledon, London SW19 8QN

Tel: (0181) 5409194 Fax: (0181) 5420494 